\section{BRAZIULIAN JOURNAL \\ OF MEDICAL AND BIOLOGICAL RESHARCH}

www.bjournal.com.br
ISSN 0100-879X

Volume 44 (1) 1-83 January 2011

CLINICAL INVESTIGATION

Braz J Med Biol Res, January 2011, Volume 44(1) 78-83

doi: 10.1590/S0100-879X2010007500122

Women with primary ovarian insufficiency have lower bone mineral density

F. Amarante, L.C. Vilodre, M.A. Maturana and P.M. Spritzer

The Brazilian Journal of Medical and Biological Research is partially financed by
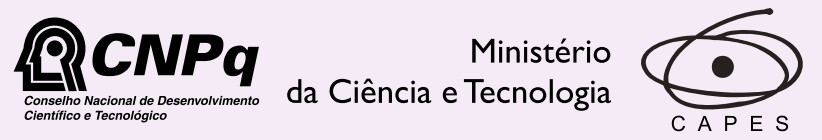

Ministério da Educação

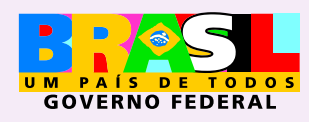

DTAPESP

Institutional Sponsors
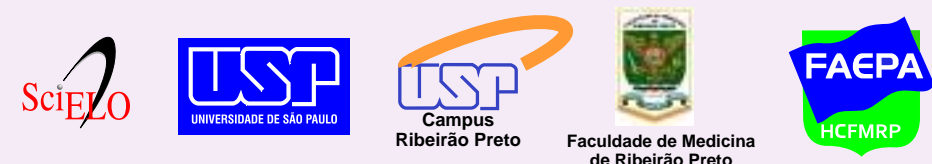

Ф SHIMADZU

GE Healthcare
Hotsite of proteomics metabolomics developped by: 


\title{
Women with primary ovarian insufficiency have lower bone mineral density
}

\author{
F. Amarante ${ }^{1}$, L.C. Vilodre ${ }^{1}$, M.A. Maturana ${ }^{1}$ and P.M. Spritzer ${ }^{1,2,3}$ \\ 1 Unidade de Endocrinologia Ginecológica, Serviço de Endocrinologia, \\ Hospital de Clínicas de Porto Alegre, Porto Alegre, RS, Brasil \\ 2Departamento de Fisiologia, Universidade Federal do Rio Grande do Sul, \\ Porto Alegre, RS, Brasil \\ ${ }^{3}$ Instituto Nacional de Hormônios e Saúde da Mulher - CNPq, \\ Universidade Federal do Rio Grande do Sul, Porto Alegre, RS, Brasil
}

\begin{abstract}
The aim of the present study was to assess the prevalence of osteoporosis in a sample of 32 patients with spontaneous primary ovarian insufficiency (POI) in comparison to reference groups of 25 pre- and 55 postmenopausal women. Hip (lumbar) and spinal bone mineral density (BMD) measurements were performed by dual-energy X-ray absorptiometry in the three groups. The median age of POI patients at the time of diagnosis was 35 years (interquartile range: $27-37$ years). The mean \pm SD age of postmenopausal reference women (52.16 \pm 3.65 years) was higher than that of $\mathrm{POI}(46.28 \pm 10.38$ years $)$ and premenopausal women (43.96 $\pm 7.08 ; \mathrm{P}=0.001)$ at the time of BMD measurement. Twenty-seven (84.4\%) POI women were receiving hormone replacement therapy (HRT) at the time of the study. In the postmenopausal reference group, $30.4 \%$ were current users of HRT. Lumbar BMD was significantly lower in the POI group $\left(1.050 \pm 0.17 \mathrm{~g} / \mathrm{cm}^{2}\right)$ compared to the age-matched premenopausal reference group (1.136 $\left.\pm 0.12 \mathrm{~g} / \mathrm{cm}^{2} ; \mathrm{P}=0.040\right)$. Moreover, $22(68.7 \%) \mathrm{POI}$ women had low bone density (osteopenia/osteoporosis by World Health Organization criteria) versus $47.3 \%$ of the postmenopausal reference group $(P=0.042)$. In conclusion, the present data indicate that BMD is significantly lower in patients with POI than in age-matched premenopausal women. Also, the prevalence of osteopenia/osteoporosis is higher in POI women than in women after natural menopause. Early medical interventions are necessary to ensure that women with POI will maintain their bone mass.
\end{abstract}

Key words: Premature ovarian failure; Premature menopause; Osteoporosis; Bone mineral density; Hypogonadism

\section{Introduction}

Primary ovarian insufficiency (POI) is defined as the premature cessation of ovarian function before 40 years of age, with findings of amenorrhea and levels of folliclestimulating hormone (FSH) in the menopausal range. POI is a fairly common condition, affecting approximately 1 in 10,000 women by age 20,1 in 1000 women by age 30 , and 1 in 100 women by age 40 (1). The causes of POI are heterogeneous $(2,3)$. Most cases are idiopathic, but acquired forms may occur after treatment for neoplastic or autoimmune diseases (3). The mechanisms underlying POI are largely unknown; however, the report of familial cases indicates a role of genetic aberrations in the pathogenesis of this disorder (4). Although genetic defects usually involve the $X$ chromosome, an increasing number of studies have documented autosomal involvement in POI (5).
POI is associated with a more prolonged low estrogen state than usually observed in natural menopause. Consequently, $\mathrm{POI}$ is potentially associated with both more severe and different health risks than natural menopause. Women entering menopause early seem to be at increased risk of cardiovascular disease (6). In addition, impaired endothelial dysfunction, an early stage of atherosclerosis, has been described in young women with POI (7).

Although early age at menopause has been reported to be associated with the development of osteoporosis (8-10), few studies so far have assessed the prevalence and factors contributing to low bone mineral density (BMD) in patients with spontaneous POI (11). Therefore, the aim of the present study was to assess the prevalence of osteoporosis in a sample of $\mathrm{POI}$ patients in comparison to

Correspondence: P.M. Spritzer, Departamento de Fisiologia, Serviço de Endocrinologia, Hospital de Clínicas de Porto Alegre, UFRGS, Rua Ramiro Barcelos, 2350, 90035-003 Porto Alegre, RS, Brasil. Fax: +55-51-3359-8777. E-mail: spritzer@ufrgs.br

Presented in part as a poster at the 8th European Congress on Menopause (EMAS), London, UK, May 2009.

Received May 26, 2010. Accepted October 20, 2010. Available online November 5, 2010. Published January 17, 2011. 
reference groups of pre- and postmenopausal women.

\section{Material and Methods}

\section{Patients and reference groups}

This controlled cross-sectional study included 32 patients with POI consulting at the Gynecological Endocrinology Unit of Hospital de Clínicas de Porto Alegre, Brazil, since March 1999 (12). Women with primary or secondary amenorrhea before the age of 40 years, a normal 46XX karyotype and $\mathrm{FSH}$ levels higher than $40 \mathrm{IU} / \mathrm{L}$ in at least two consecutive measurements were selected. Patients with secondary causes of POI, such as surgery, chemotherapy or radiotherapy, and chromosomal abnormalities were excluded. The study protocol was approved by the Ethics Committee of Hospital de Clínicas de Porto Alegre and written informed consent was obtained from each subject.

Two reference groups were also included: a group of 25 premenopausal women matched for age, and a group of 55 postmenopausal women matched for menopausal status. All had undergone evaluation of BMD using dualenergy X-ray absorptiometry densitometer (DXA) between September and November 2007. Postmenopause was defined as one year or more of amenorrhea after the age of 40 years. Patients with thyroid dysfunction were excluded. All patients in the reference groups also gave verbal informed consent.

\section{Study protocol}

Anthropometric measurements included body weight, height, and body mass index (BMI, current weight in $\mathrm{kg}$ divided by height in $\mathrm{m}^{2}$ ). Hormonal and biochemical variables were assessed in POI patients on any day after an overnight fast. Blood samples were drawn from an antecubital vein for determination of serum $\mathrm{FSH}$, estradiol and thyroid-stimulating hormone (TSH). All samples were obtained between 8:00 and 10:00 am. inter-assay CV of 8.6 and $2.83 \%$, respectively.

\section{Measurement of BMD}

BMD was measured in all subjects by the same person (FA). Measurements obtained at both the lumbar spine (L1-L4) and total femoral hip levels were analyzed by DXA (Lunar Radiation Corporation, DPX, USA) in a private clinic (SIDI, Brazil). As established by the International Society for Clinical Densitometry, the CV was $1.46 \%$ for the lumbar spine and $0.90 \%$ for the femur. World Health Organization (WHO) criteria were used to categorize bone mass status (osteopenia: T scores between -1 and -2.5 SD below the average for the reference population; osteoporosis: $T$ scores of $\geq-2.5 \mathrm{SD}$ for a young adult reference population) (13). Normal bone mass was defined as a T score $\leq-1.0$ SD and low bone mass was defined as the presence of at least one site of osteopenia or osteoporosis.

\section{Statistical analysis}

Data are reported as means \pm SD or median and interquartile range (IQR: $25-75 \%)$. The Student $t$-test was used for comparisons between two group means and the Mann-Whitney U-test was used for comparisons between two group medians. Comparisons between three groups were analyzed by one-way ANOVA or the Kruskal-Wallis $\mathrm{H}$ test using a two-tailed test for variables with a Gaussian or non-Gaussian distribution, respectively. Pearson's correlation coefficient was calculated for BMD vs BMI in the POI group. Comparisons between ratios were carried out using the $X^{2}$ test. All analyses were performed using the Statistical Package for the Social Sciences (SPSS, USA). Data were considered to be significant at $P<0.05$.

\section{Results}

The median age of $\mathrm{POI}$ patients at the time of diagnosis was 35 years (IQR: 27-37 years). Table 1 shows the mean age of POI patients and reference groups at the time of

\begin{abstract}
Assays
Serum FSH was measured by electrochemiluminescence immunoassay (ECLIA; Elecsys 2010, Roche Diagnostics, Germany) with intra- and inter-assay coefficients of variation (CV) of 4.8 and $3.3 \%$, respectively. The sensitivity of the assay was 0.05 IU/L for FSH. Estradiol was measured by ECLIA (Elecsys 2010), with an assay sensitivity of 5.0 $\mathrm{pg} / \mathrm{mL}$, and with intra- and interassay $C V$ of 5.7 and $6.4 \%$. Serum TSH was measured by ECLIA (Elecsys 2010) with intra- and
\end{abstract}

Table 1. Characteristics of women with primary ovarian insufficiency and reference groups at the time of dual-energy X-ray absorptiometry.

\begin{tabular}{lccc}
\hline & POI $(\mathrm{N}=32)$ & \multicolumn{2}{c}{ Reference groups } \\
\cline { 3 - 4 } & & $\begin{array}{c}\text { Premenopausal } \\
(\mathrm{N}=25)\end{array}$ & $\begin{array}{c}\text { Postmenopausal } \\
(\mathrm{N}=55)\end{array}$ \\
\hline Age (years) & $46.28 \pm 10.38^{*}$ & $43.96 \pm 7.08^{*}$ & $52.16 \pm 3.65$ \\
BMI & $24.98 \pm 4.77$ & $25.80 \pm 5.25$ & $27.00 \pm 5.01$ \\
Age at menarche (years) & $12.90 \pm 2.51$ & $12.08 \pm 1.41$ & $12.41 \pm 1.69$ \\
Users of HRT at the time of DXA & $27(84.4 \%)^{+}$ & - & $17(30.4 \%)$ \\
\hline
\end{tabular}

Data are reported as means $\pm S D\left({ }^{*} P=0.001\right.$ compared to postmenopausal reference group, ANOVA) or as number and percentage $\left({ }^{+} P<0.001\right.$ compared to postmenopausal reference group, chi-square test). $\mathrm{POI}=$ primary ovarian insufficiency; $\mathrm{BMI}=$ body mass index; HRT = hormone replacement therapy; DXA = dual-energy X-ray absorptiometry. 
DXA examination. The mean age of women in the postmenopausal reference group was higher than that of the $\mathrm{POI}$ and premenopausal groups $(P=0.001)$. Except for 2 patients in the $\mathrm{POI}$ group and 1 in the reference group who were of mixed (African and European) descent, all patients were Caucasians. $\mathrm{BMI}$ and age at menarche were similar in all groups.

Table 2. Lumbar and femoral bone mineral density in the primary ovarian insufficiency and postmenopausal reference groups.

\begin{tabular}{lcc}
\hline & POI $(\mathrm{N}=32)$ & $\begin{array}{c}\text { Postmenopausal reference } \\
\text { group }(\mathrm{N}=55)\end{array}$ \\
\hline L1-L4 BMD $\left(\mathrm{g} / \mathrm{cm}^{2}\right)$ & $1.050 \pm 0.17$ & $1.078 \pm 0.02$ \\
Femur BMD $\left(\mathrm{g} / \mathrm{cm}^{2}\right)$ & $0.919 \pm 0.13$ & $0.944 \pm 0.13$ \\
\hline
\end{tabular}

Data are reported as means $\pm S D$. No statistical differences were detected (Student $t$-test). $\mathrm{POI}=$ primary ovarian insufficiency; $\mathrm{BMD}=$ bone mineral density.
Twenty-seven (84.4\%) POI women had sought medical advice previously and had taken a variety of estrogen and progestin regimens. The median time elapsed between the beginning of hypoestrogenic symptoms and the start of hormone therapy was 3 years, ranging from 2 to 5 years. In the postmenopausal reference group, 17 women (30.4\%) were current users of hormone therapy.

BMD data are presented in Table 2 and Figure 1. No differences in femur BMD were observed between the POI group and the postmenopausal reference group (Table 2). In contrast, lumbar BMD was lower in the POI group compared to the age-matched premenopausal reference group (Figure 1).

A positive correlation was observed between BMI and femur BMD in all groups (Table 3). Concerning the lumbar site, a positive correlation was found only in the postmenopausal reference group.

Table 4 shows that the prevalence of low BMD differed significantly between the POI and postmenopausal groups.

Table 3. Correlations between lumbar (L1-L4) and femoral bone mineral density and body mass index in the primary ovarian insufficiency and reference groups.

\begin{tabular}{|c|c|c|c|c|c|c|}
\hline & \multirow{2}{*}{\multicolumn{2}{|c|}{$\mathrm{POI}(\mathrm{N}=32)$}} & \multicolumn{4}{|c|}{ Reference group } \\
\hline & & & \multicolumn{2}{|c|}{ Premenopausal $(\mathrm{N}=25)$} & \multicolumn{2}{|c|}{ Postmenopausal $(\mathrm{N}=55)$} \\
\hline & L1-L4 & Femur & L1-L4 & Femur & L1-L4 & Femur \\
\hline $\operatorname{BMD}\left(\mathrm{g} / \mathrm{cm}^{2}\right) v s$ & $r=-0.001$ & $r=0.522$ & $r=0.303$ & $r=0.641$ & $r=0.271$ & $r=0.607$ \\
\hline BMI $\left(\mathrm{kg} / \mathrm{m}^{2}\right)$ & $P=0.994$ & $P=0.002$ & $P=0.140$ & $P=0.001$ & $P=0.043$ & $P<0.001$ \\
\hline
\end{tabular}

$r$ = Pearson correlation coefficient; $\mathrm{POI}=$ primary ovarian insufficiency; $\mathrm{BMD}=$ bone mineral density; $\mathrm{BMI}=$ body mass index; Femur $=$ femoral $\mathrm{BMD}$ at the hip; L1-L4 $=\mathrm{BMD}$ at the lumbar spine.
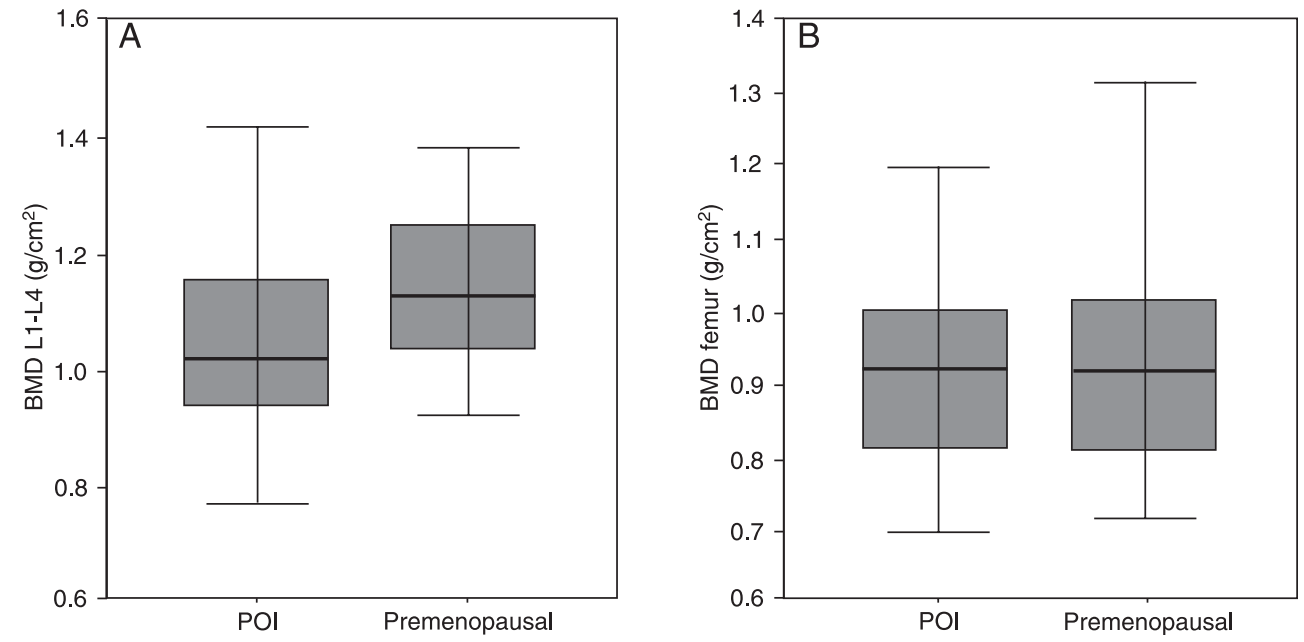

Figure 1. Bone mineral density (BMD) of the primary ovarian insufficiency $(\mathrm{POI}, \mathrm{N}=32$ ) and premenopausal reference $(\mathrm{N}=25)$ groups. $A, \mathrm{~L} 1$-L4 lumbar bone mineral density ( ${ }^{*} \mathrm{P}=0.040$; Student $t$-test). $B$, Femoral bone mineral density. 
Table 4. Prevalence of low bone mineral density in the primary ovarian insufficiency and postmenopausal reference groups.

\begin{tabular}{lcc}
\hline & Normal BMD & Low BMD \\
\hline POI $(\mathrm{N}=32)$ & $10(31.3 \%)$ & $22(68.7 \%)^{*}$ \\
Postmenopausal $(\mathrm{N}=55)$ & $29(52.7 \%)$ & $26(47.3 \%)$ \\
\hline
\end{tabular}

$\mathrm{BMD}=$ bone mineral density; Low BMD = lumbar and/or femur osteopenia or osteoporosis; $\mathrm{POI}=$ primary ovarian insufficiency. ${ }^{*} \mathrm{P}=0.042$ compared to postmenopausal group (chi-square test).

Table 5. Age at menopause, body mass index and hormonal profile of women with primary ovarian insufficiency, stratified by bone mineral density.

\begin{tabular}{lcc}
\hline & $\begin{array}{c}\text { Normal BMD } \\
(\mathrm{N}=10)\end{array}$ & $\begin{array}{c}\text { Low BMD } \\
(\mathrm{N}=22)\end{array}$ \\
\hline Age at last menses (years) & $34 \pm 4.6$ & $30 \pm 9$ \\
$\mathrm{BMI}$ & $24 \pm 3.3$ & $24 \pm 4.4$ \\
$\mathrm{FSH}(\mathrm{IU} / \mathrm{L})$ & $56(32-139)$ & $52(6-170)$ \\
$\mathrm{TSH}(\mathrm{mlU} / \mathrm{L})$ & $3.15(1.32-4.08)$ & $1.90(1.34-2.98)$ \\
Estradiol $(\mathrm{pM})$ & $86(18-200)$ & $39(18-71)$ \\
\hline
\end{tabular}

Data are reported as means \pm SD (ANOVA) or median and interquartile range (Kruskal-Wallis test). No statistical differences were detected. $\mathrm{BMD}=$ bone mineral density; $\mathrm{BMI}=$ body mass index; $\mathrm{FSH}=$ follicle-stimulating hormone; $\mathrm{TSH}=$ thyroid-stimulating hormone; Low BMD = lumbar and/or femur osteopenia or osteoporosis.

Sixty-nine percent of POI women were found to have low BMD (osteopenia/osteoporosis by WHO criteria) vs $47 \%$ in the postmenopausal reference group $(P=0.042)$.

Table 5 shows the age at last period, BMI and hormonal profile of POI patients, stratified by normal or low BMD. Age at menopause and BMI were similar in POI patients with normal and low BMD. There was no significant difference in serum $\mathrm{FSH}$, TSH, or estradiol levels between POI patients stratified by normal or low bone mass.

\section{Discussion}

In the present study, women with primary ovarian insufficiency had lower BMD than premenopausal women of similar age and a higher prevalence of osteopenia/osteoporosis than women after natural menopause. Although the risk of osteoporosis in patients with $\mathrm{POI}$ is a source of concern, few studies have compared POI women with two simultaneous reference groups. Uygur et al. (9) reported lower BMD in POI patients than in a control group of normal women of similar age. Hadjidakis et al. (14) compared bone mass in four groups: early menopause, surgical menopause and two natural menopause groups. Women entering menopause precociously exhibited lower vertebral BMD than women of similar age after either surgical or natural menopause.

In our study, the comparison of BMD between the POI and reference groups was performed taking into consideration the age when densitometry was performed. For some patients in the $\mathrm{POI}$ or postmenopause reference group, BMD was determined many years after the last period/diagnosis of $\mathrm{POI}$. We observed that $\mathrm{POI}$ patients had significantly lower BMD than the age-matched premenopausal control group. In addition, the prevalence of low bone density was higher in the POI group than in the postmenopausal control group, even though the latter women were significantly older. These data suggest a strong impact of decreased estrogen levels on bone mass in women before the age of 40 years.

In the present study, lower BMD was observed mainly in the lumbar spine of POI patients. This was probably due to the greater impact of hypoestrogenic status on trabecular bone (15) and is in accordance with previous studies (16).

Chronological age and time since menopause negatively affect bone density in women of similar age in surgical or natural menopause (14). In the present study, $68 \%$ of the POI group had low bone mass, after a median of 4 years of amenorrhea. These data agree with the results of studies reporting that early menopause, whether natural or surgical, is an established risk factor for osteoporosis $(9,14,17-19)$. The prevalence of osteoporosis increases markedly after the age of menopause, and the concept that estrogen deficiency is critical to the pathogenesis of osteoporosis was initially based on the fact that postmenopausal women, whose estrogen levels naturally decline, are at highest risk to develop the disease (20). Morphologic studies and measurements of certain biochemical markers have indicated that bone remodeling is accelerated during menopause, since the markers of both bone resorption and formation are increased (21). Although it is well known that estrogen has direct effects on bone cells, recent animal studies have identified additional unexpected regulatory effects of estrogen on adaptive immune response and oxidative stress. These mechanisms seem to be implicated in the pathogenesis of osteoporosis $(22,23)$. Changes in cytokine production within bone marrow, as well as proapoptotic and anti-apoptotic effects on osteoblastic cells have been proposed as possible cellular and molecular mechanisms by which sex steroids influence adult bone homeostasis (24).

The use of estrogen therapy has been positively associated with amelioration of BMD in postmenopausal women, and hormone replacement therapy remains a valuable option for the prevention and treatment of osteoporosis in normal early postmenopausal women $(10,14,25-27)$. 
The postmenopausal estrogen-progestin interventions trial (PEPI trial) also showed that estrogen-progestin replacement therapy could maintain bone mass in postmenopausal women aged $45-64$ years (28).

In turn, although our patients had previously sought medical advice and most of them had taken a variety of estrogen and progestin replacement regimens at least intermittently, they still had reduced BMD compared to women of similar age with normal ovarian function. It is clear that these young women need ongoing medical supervision and education regarding the need for hormone replacement, physical activity, and adequate calcium and vitamin D supplementation. Furthermore, strategies to improve compliance are needed.

Several studies have found that increased BMI and weight are associated with slower rates of peri- and postmenopausal bone loss $(14,15,29)$. In the present study, this positive association between BMD and BMI was confirmed in all groups, i.e., POI, pre- and postmenopausal women. While the precise mechanisms for this association are still unclear, some possibilities have been raised. Body weight affects both bone turnover and bone density, and is therefore as important as age as a risk factor for vertebral and hip fractures. A number of mechanisms for the fatbone relationship exist and include the effect of soft tissue mass on skeletal loading, the association of fat mass with the secretion of bone-active hormones from pancreatic $\beta$ cells (including insulin, amylin, and preptin), and the secretion of bone-active hormones (e.g., estrogens and leptin) from adipocytes (30). Recent experimental findings have shown the involvement of adipokines, including leptin and adiponectin, in bone metabolism $(31,32)$. However, contrasting studies suggest that excessive fat mass may not protect against osteoporosis or osteoporotic fracture.

\section{References}

1. Coulam CB, Adamson SC, Annegers JF. Incidence of premature ovarian failure. Obstet Gynecol 1986; 67: 604-606.

2. Vilodre LC, Moretto M, Kohek MB, Spritzer PM. [Premature ovarian failure: present aspects]. Arq Bras Endocrinol Metabol 2007; 51: 920-929.

3. Santoro N. Mechanisms of premature ovarian failure. Ann Endocrinol 2003; 64: 87-92.

4. Conway GS. Premature ovarian failure. Curr Opin Obstet Gynecol 1997; 9: 202-206.

5. Nippita TA, Baber RJ. Premature ovarian failure: a review. Climacteric 2007; 10: 11-22.

6. Kok HS, van Asselt KM, van der Schouw YT, van der Tweel I, Peeters PH, Wilson PW, et al. Heart disease risk determines menopausal age rather than the reverse. J Am Coll Cardiol 2006; 47: 1976-1983.

7. Kalantaridou SN, Naka KK, Papanikolaou E, Kazakos N, Kravariti M, Calis KA, et al. Impaired endothelial function in young women with premature ovarian failure: normalization with hormone therapy. J Clin Endocrinol Metab 2004; 89:
Differences in experimental design, sample structure, and even the selection of covariates may account for some of these inconsistent or contradictory results (33).

Our findings may have important clinical implications. In this sample of $\mathrm{POI}$ patients with a short time since amenorrhea, $68 \%$ presented low bone mass, suggesting that the bone loss began to occur before the cycles were interrupted, or else that the patients did not achieve their peak bone mass. This is in accordance with longitudinal studies of women entering menopause naturally, and suggests that bone loss may start in the premenopausal years $(29,34,35)$ and that screening for BMD should be performed in the presence of menstrual irregularities in young women with suspected diagnosis of $\mathrm{POI}$ or risk factors for this disorder.

While presenting significant data on the bone mass status of $\mathrm{POI}$ women, our study has a limitation related to the fact that patients in the pre- and postmenopausal control groups were included in the study as a convenience sample. Therefore, laboratory data could not be obtained for these groups.

The results of the present study show that women with karyotypically normal spontaneous POI have a significantly lower BMD than normal women. These data indicate that hormone replacement therapy should be prescribed early and consistently to affected patients. Young women at risk for $\mathrm{POI}$ need early education regarding strategies to maintain bone mass and ongoing medical supervision to ensure compliance with these strategies.

\section{Acknowledgments}

Research supported by CNPq and Fundo de Incentivo à Pesquisa HCPA (FIPE).
3907-3913.

8. Anasti JN, Kalantaridou SN, Kimzey LM, Defensor RA, Nelson LM. Bone loss in young women with karyotypically normal spontaneous premature ovarian failure. Obstet Gynecol 1998; 91: 12-15.

9. Uygur D, Sengul O, Bayar D, Erdinc S, Batioglu S, Mollamahmutoglu L. Bone loss in young women with premature ovarian failure. Arch Gynecol Obstet 2005; 273: 17-19.

10. Metka M, Holzer G, Heytmanek G, Huber J. Hypergonadotropic hypogonadic amenorrhea (World Health Organization III) and osteoporosis. Fertil Steril 1992; 57: 37-41.

11. Popat VB, Calis KA, Vanderhoof VH, Cizza G, Reynolds JC, Sebring $\mathrm{N}$, et al. Bone mineral density in estrogen-deficient young women. J Clin Endocrinol Metab 2009; 94: 22772283.

12. Vilodre LC, Kohek MB, Spritzer PM. Screening of folliclestimulating hormone receptor gene in women with premature ovarian failure in southern Brazil and associations with phenotype. J Endocrinol Invest 2008; 31: 552-557. 
13. Kanis JA, Melton LJ III, Christiansen C, Johnston CC, Khaltaev N. The diagnosis of osteoporosis. J Bone Miner Res 1994; 9: 1137-1141.

14. Hadjidakis D, Kokkinakis E, Sfakianakis M, Raptis SA. The type and time of menopause as decisive factors for bone mass changes. Eur J Clin Invest 1999; 29: 877-885.

15. Guthrie JR, Ebeling PR, Hopper JL, Barrett-Connor E, Dennerstein L, Dudley EC, et al. A prospective study of bone loss in menopausal Australian-born women. Osteoporos Int 1998; 8: 282-290.

16. Bagur AC, Mautalen CA. Risk for developing osteoporosis in untreated premature menopause. Calcif Tissue Int 1992; 51: 4-7.

17. Pouilles JM, Tremollieres F, Bonneu M, Ribot C. Influence of early age at menopause on vertebral bone mass. J Bone Miner Res 1994; 9: 311-315.

18. Shilbayeh S. Prevalence of osteoporosis and its reproductive risk factors among Jordanian women: a cross-sectional study. Osteoporos Int 2003; 14: 929-940.

19. Gokmen O, Seckin NC, Sener AB, Ozaksit G, Ekmekci S. A study of premature ovarian failure in Turkish women. Gynecol Endocrinol 1995; 9: 283-287.

20. Albright F, Blomberg F, Smith PH. Postmenopausal osteoporosis. Trans Assoc Am Physicians 1940; 55: 298-305.

21. Ebeling PR, Atley LM, Guthrie JR, Burger HG, Dennerstein L, Hopper JL, et al. Bone turnover markers and bone density across the menopausal transition. J Clin Endocrinol Metab 1996; 81: 3366-3371.

22. Weitzmann MN, Pacifici R. Estrogen deficiency and bone loss: an inflammatory tale. J Clin Invest 2006; 116: 11861194.

23. Raisz LG. Pathogenesis of osteoporosis: concepts, conflicts, and prospects. J Clin Invest 2005; 115: 3318-3325.

24. Balasch J. Sex steroids and bone: current perspectives. Hum Reprod Update 2003; 9: 207-222.

25. Cauley JA, Robbins J, Chen Z, Cummings SR, Jackson RD, LaCroix AZ, et al. Effects of estrogen plus progestin on risk of fracture and bone mineral density: the Women's Health Initiative randomized trial. JAMA 2003; 290: 1729-1738.

26. Jackson RD, Wactawski-Wende J, LaCroix AZ, Pettinger
M, Yood RA, Watts NB, et al. Effects of conjugated equine estrogen on risk of fractures and BMD in postmenopausal women with hysterectomy: results from the women's health initiative randomized trial. J Bone Miner Res 2006; 21: 817828.

27. Siris ES, Miller PD, Barrett-Connor E, Faulkner KG, Wehren LE, Abbott TA, et al. Identification and fracture outcomes of undiagnosed low bone mineral density in postmenopausal women: results from the National Osteoporosis Risk Assessment. JAMA 2001; 286: 2815-2822.

28. Effects of hormone therapy on bone mineral density: results from the postmenopausal estrogen/progestin interventions (PEPI) trial. The Writing Group for the PEPI. JAMA 1996; 276: 1389-1396.

29. Finkelstein JS, Brockwell SE, Mehta V, Greendale GA, Sowers MR, Ettinger B, et al. Bone mineral density changes during the menopause transition in a multiethnic cohort of women. J Clin Endocrinol Metab 2008; 93: 861-868.

30. Reid IR. Relationships among body mass, its components, and bone. Bone 2002; 31: 547-555.

31. Nishimura R, Hata K, Yoneda T. [Relationship between bone metabolism and adipogenesis]. Clin Calcium 2007; 17: 233240.

32. Zoico E, Zamboni M, Di Francesco V, Mazzali G, Fantin F, De Pergola G, et al. Relation between adiponectin and bone mineral density in elderly postmenopausal women: role of body composition, leptin, insulin resistance, and dehydroepiandrosterone sulfate. J Endocrinol Invest 2008; 31: 297-302.

33. Zhao LJ, Jiang H, Papasian CJ, Maulik D, Drees B, Hamilton $\mathrm{J}$, et al. Correlation of obesity and osteoporosis: effect of fat mass on the determination of osteoporosis. $J$ Bone Miner Res 2008; 23: 17-29.

34. Pal L, Bevilacqua K, Zeitlian G, Shu J, Santoro N. Implications of diminished ovarian reserve (DOR) extend well beyond reproductive concerns. Menopause 2008; 15: 10861094.

35. Falch JA, Sandvik L. Perimenopausal appendicular bone loss: a 10-year prospective study. Bone 1990; 11: 425-428. 